\title{
Nanofiltration membranes of poly(styrene-co-chloro- methylstyrene)-grafted-DGEBA reinforced with gold and polystyrene nanoparticles for water purification
}

\author{
Ayesha Kausar ${ }^{1} \cdot$ Muhammad Siddiq $^{2}$
}

Received: 26 August 2014/Accepted: 20 September 2015/Published online: 3 October 2015

(c) The Author(s) 2015. This article is published with open access at Springerlink.com

\begin{abstract}
The matrix material for nanofiltration membranes was prepared through chemical grafting of poly (styrene-co-chloromethylstyrene) (PSCMS) to DGEBA using hexamethylenediamine as linker. The phase inversion technique was used to form PSCMS- $g$-DGEBA membranes. This effort also involves the designing of gold nanoparticles and its composite nanoparticles with polystyrene microspheres as matrix reinforcement. The nanoporous morphology was observed at lower filler content and there was formation of nanopattern at increased nanofiller content. The tensile strength was improved from 32.5 to $35.2 \mathrm{MPa}$ with the increase in AuNPs-PSNPs loading from 0.1 to $1 \mathrm{wt} \%$. The glass transition temperature was also enhanced from 132 to $159{ }^{\circ} \mathrm{C}$. The membrane properties were measured via nanofiltration set-up. Higher pure water permeation flux, recovery, and salt rejection were measured for novel membranes. PSCMS- $g$-DGEBA/ AuNPs-PSNPs membrane with $1 \mathrm{wt} \%$ loading showed flux of $2.01 \mathrm{~mL} \mathrm{~cm}^{-2} \mathrm{~min}^{-1}$ and salt rejection ratio of $70.4 \%$. Efficiency of the gold/polystyrene nanoparticles reinforced membranes for the removal of $\mathrm{Hg}^{2+}$ and $\mathrm{Pb}^{2}$ was found to be $99 \%$. Novel hybrid membranes possess fine characteristics to be utilized in industrial water treatment units.
\end{abstract}

Ayesha Kausar

asheesgreat@yahoo.com

1 Nanosciences and Catalysis Division, National Centre For Physics, Quaid-i-Azam University Campus, Islamabad 44000, Pakistan

2 Department of Chemistry, Quaid-i-Azam University, Islamabad, Pakistan
Keywords Nanofiltration P Poly(styrene-cochloromethylstyrene) - Epoxy · Phase inversion . Pure water flux

\section{Introduction}

The membrane segregation processes are extensively employed for the water treatment in various industries such as chemical, food, nuclear, and pharmaceutical productions. The membrane processes offer an attractive alternate to the traditional systems for the ultra pure water production, desalination, pathogen exclusion from water, and solid-liquid isolation in water treatment. The membrane technology is an attractive method in water purification industries (Arockiasamy et al. 2013). The membrane separation process has high functional stability and is easy to control and integrate in larger industrial practices (Kumar et al. 2013). The important types of membrane processes presently employed are ultrafiltration (UF), nanofiltration (NF), and reverse osmosis (RO). Generally, the RO technique is based on salt ion repellent capacity of thick membrane, so, allowing the flow of water molecules (Greenlee et al. 2009). The process is usually controlled through solution-diffusion with a sufficiently large external pressure to overcome the salt water osmotic pressure (Evuti and Lawal 2011). On the other hand, UF membranes permit the separation of intermediate molecular weight pollutants and certain heavy metals. The effectiveness of both the membrane processes depends on their design and properties (Yuan and Zydney 2000). Among them, NF technique is known for smaller pore size of membrane and optimal purification efficacy (Dalwani et al. 2010). At present, there are two extensively used categories of membranes for water detoxification, i.e., polymeric 
membranes (polysulfone, polyamide, polyfurane, etc.), and ceramic/inorganic composite (Bowen et al. 1997; Chen et al. 2001). The polymeric membranes are of primary significance due to their flexibility and low costs. However, these membranes experience the lack of thermal and chemical stability. In this regard, the hybrid polymeric membranes are well established in desalination due to straightforward module design, improved selectivity, and permeability (Bowen and Mohammad 1998; Bowen and Welfoot 2002). The desalination operates through various transference mechanisms depending mostly on the membrane pore sizes. The hybrid membranes are quite robust to process conditions such as long-term stability at elevated temperature under hydrothermal conditions, continuous operation over prolonged time periods without any decline of selectivity, and acid stability of the material. Therefore, the beneficial use of hybrid polymer/inorganic materials has been exploited in membrane technology (Gevers et al. 2005). In this area, various inorganic fillers such as titania (Ebert et al. 2004), silica (Sadeghi et al. 2008), and carbon molecular sieves ( $\mathrm{Vu}$ et al. 2003) were employed to fabricate hybrid thin films. For the removal of pollutants from ground water, hybrid nanofiltration seems to be a suitable method capable of removing hardness, sulfates, chlorides, and bacteria.

Removal of heavy metals is an essential area of research in membrane filtration technology. An important property presented by modified surfaces of noble metal nanoparticles is the diagnosis of heavy metals. Initial studies regarding the interaction between metal ions and noble metal nanoparticles were carried out in 1990s (Henglein 1998). A number of adsorbents have been industrialized for the elimination of mercury from water such as activated carbon (Namasivayam and Periasamy 1993) and functionalized clay (Asyhar 2014). Likewise, there is literature available regarding the nanomaterial-based mercury elimination from contaminated water ( $\mathrm{Lu}$ and Liu 2007). One of these methods involves the use of biomolecule-modified gold nanoparticles (Ono and Togashi 2004). In a comparable attempt, the peptide-modified gold nanoparticles were used for colorimetric recognition of $\mathrm{Pb}^{2+}$ and $\mathrm{Hg}^{2+}$ (Slocik et al. 2008). However, the hybrid nanofiltration membrane has been less explored in literature compared with other membranes types. The important factors affecting the morphology and performance of membranes are the type and the concentration of nanofiller (Ebert et al. 2004). In this exploration, the matrix poly(styrene-co-chloromethylstyrene) was chemically grafted to epoxy (bisphenol A diglycidyl ether) using hexamethylenediamine (PSCMS- $g$ DGEBA). The phase inversion technique was used to form nanofiltration membranes loaded with gold/polystyrene composite nanoparticle (AuNPs-PSNPs). The noble metal nanoparticles were introduced as reinforcement to remove several toxic metal ions from water. The influence of the inclusion of various AuNPs-PSNP contents on membrane performance and structure was studied using pertinent methods and techniques. To the best of our knowledge, epoxy blend membranes of poly(styrene-co-chloromethylstyrene)-grafted-DGEBA have been prepared for the first time. Novelty of the research also lies in the reinforcement of the membranes with the gold composite nanoparticles. Epoxy has been employed in water filtration membranes (Du et al. 2011). In this research, the cured DGEBA was first blended with poly(styrene-co-chloromethylstyrene). Afterwards, the composite membranes of poly(styrene-co-chloro-methylstyrene)-grafted-DGEBA with the nanoparticles were formed. After blending of the composite nanoparticles in cured epoxy blend structure, there are least chances of the gold nanoparticles to move into the media relative to reported gold nanoparticlepolymer composites (Coulston et al. 2011). The fabrication strategy opted for the AuNPs-PSNPs resulted in fine morphology compared with the other reported methods ( $\mathrm{Li}$ et al. 2011). The spherical shape and high surface area of AuNPs-PSNPs increased their compatibility with the nanofiller. The membranes simply formed by poly(styreneco-chloro-methylstyrene)-grafted-DGEBA may have lower mechanical properties. Therefore, the nanoparticles were used as matrix component to increase the tensile and other physical properties of the hybrids (Susrutha et al. 2012). The mechanical properties of the hybrid membranes were superior to that of the gold-reinforced chitosan bionanocomposites (Patel et al. 2014).

\section{Experimental}

\section{Materials}

Poly(styrene-co-chloromethylstyrene $) \quad\left(M_{\mathrm{w}} \sim 100,000\right)$, was purchased from Aldrich. Styrene ( $\geq 99 \%)$, obtained from Aldrich was purified by distillation under reduced pressure. Poly(vinylpyrrolidone) (PVP K-30) was purchased from Acros Organics. Bisphenol A diglycidyl ether (DGEBA, $\geq 95.0 \%$ ), hexamethylenediamine (HDA, $98 \%$ ), 2-2'-azoisobutyronitrile (AlBN, $\geq 98 \%$ ), $\mathrm{HAuCl}_{4}$ $(10 \mathrm{~nm}$ particle size), 4-(4-aminophenylsulfonyl)benzenamine (APSBA) (97\%), ammonium thiocyanate (98\%), and sodium carbonate were obtained from Aldrich and used as received.

\section{Characterization techniques}

The infrared (IR) spectra were recorded using a Fourier transform infrared (FTIR) Spectrometer, Model No. FTSW 300 MX, manufactured by BIO-RAD, California, USA 
( $4 \mathrm{~cm}^{-1}$ resolution). PerkinElmer's LAMBDA $45 \mathrm{UV} / \mathrm{V}$ is system was used to detect gold nanoparticles in the filtrate. The morphology, size, and distribution of AuNPs-PSNPs were deliberated through transmission electron microscopy. Scanning electron microscopy was employed to study the cross-section and internal fractured surface structure of PSCMS- $g$-DGEBA/AuNPs-PSNPs membranes. The SEM images were obtained by Scanning Electron Microscope S-4700 (Japan Hitachi Co. Ltd.) and TEM images were taken by Transmission Electron Microscope H-800 (Japan Hatachi Co. Ltd). The particle size and the particle size distribution were measured by Zetasizer Nano ZS particle analyzer (UK Malvern Instruments Ltd.) by means of dynamic light scattering (DLS). Brunauer Emmett Teller (BET) surface area measurement of membranes was carried out using a Micromeritics ASAP (Accelerated Surface Area and Porosimetry) 2000, (Micromeritics Instruments, Co., Georgia, USA). The measurement of surface area with this instrument was based on physisorption and desorption of a gas (nitrogen) at the surface and in the pores of the sample. The amount of gas adsorbed was calculated from measurement of differential gas pressure. Before analysis, the membrane samples were prepared by degassing under vacuum at $30{ }^{\circ} \mathrm{C}$ for $2 \mathrm{~h}$. The tensile tests were conducted with dog-bone-shape samples on a Testomeric materials testing machine M500-30CT at a speed of $500 \mathrm{~mm} / \mathrm{min}$ according to ASTM D412. To analyze the water content, membranes were soaked in distilled water for $24 \mathrm{~h}$ followed by mopping with blotting paper and weighing. The wet membranes were first kept at $100{ }^{\circ} \mathrm{C}$ for $24 \mathrm{~h}$, and subsequently weighed to obtain the dry weight using an electronic balance. The percent solvent content was evaluated using the following equation:

$\%$ Solvent content $=\frac{W_{1}-W_{2}}{W_{2}} \times 100$,

where $W_{1}=$ Wet membrane weight $(\mathrm{g})$ and $W_{2}=$ Dry membrane weight $(\mathrm{g})$.

To analyze the membrane porosity, samples were soaked in distilled water for $24 \mathrm{~h}$ followed by mopping with blotting paper and weighing. The membranes were dried at $100{ }^{\circ} \mathrm{C}$ for $24 \mathrm{~h}$ and weighed. Porosity was calculated using the following equation:

Porosity $=\frac{W_{1}-W_{2}}{A_{\mathrm{h}}}$,

where $W_{1}=$ Wet membrane weight $(\mathrm{g}), W_{2}=$ Dry membrane weight $(\mathrm{g})$, and $A_{\mathrm{h}}=$ Membrane area $\left(\mathrm{cm}^{2}\right)$.

Pure water flux of membrane was measured using membrane cell with cross-flow mode. The permeate was collected every $10 \mathrm{~min}$ for $1 \mathrm{~h}$. Membranes after compaction were subjected to pure water flux estimation at trans-membrane pressure of $345 \mathrm{kPa}$. The permeability was measured under steady-state flow. Pure water flux was calculated as follows:

$J_{\mathrm{w}}=\frac{Q}{A t}$,

where $Q=$ Quantity of permeate collected (L), $J_{\mathrm{w}}=$ Pure water flux (mLcm $\left.{ }^{-2} \mathrm{~min}^{-1}\right), A=$ Membrane area $\left(\mathrm{cm}^{-2}\right)$, $t=$ Sampling time $(\mathrm{min})$.

The length and width of a piece of wet membrane, immersed in a water bath, were measured using a vernier caliper. Then the membrane was heated at $100{ }^{\circ} \mathrm{C}$ for $24 \mathrm{~h}$. The dry membrane's length and width were measured. The shrinkage ratio was calculated using the following equation:

Shrinkage ratio $=\left[1-\left(\frac{a b}{a_{\mathrm{o}} b_{\mathrm{o}}}\right)\right] \times 100$,

where $A=$ length of dry membrane (m), $b=$ breadth of dry membrane $(\mathrm{m}), a_{\mathrm{o}}=$ length of wet membrane $(\mathrm{m})$, $b_{\mathrm{o}}=$ breadth of wet membrane $(\mathrm{m})$.

Rejection indicates the amount of components rejected by membranes. It shows the separation efficiency of the components by the membrane, which is calculated as

$R_{e}=1-\frac{C_{\mathrm{p}}}{C_{\mathrm{f}}}$,

where $R_{\mathrm{e}}=$ Rejection, $C_{\mathrm{p}}=$ Concentration of components in permeate $\left(\mathrm{g} / \mathrm{m}^{3}\right), C_{\mathrm{f}}=$ Concentration of components in feed water $\left(\mathrm{g} / \mathrm{m}^{3}\right)$.

Recovery is also known as productivity. Here also the membrane cell with cross-flow mode was used. According to mass balance, the feed flow is equal to the sum of concentrate flow and permeates flow. Recovery was calculated as follows:

$\gamma=\frac{Q_{\mathrm{p}}}{Q_{\mathrm{f}}} \times 100$

where $\Gamma=$ Recovery $(\%), Q_{\mathrm{p}}=$ Permeate flow $\left(\mathrm{m}^{3} / \mathrm{h}\right)$, $Q_{\mathrm{f}}=$ Feed flow $\left(\mathrm{m}^{3} / \mathrm{h}\right)$.

The solid-liquid extraction tests of nitrates or chloride salts of $\mathrm{Fe}^{3+}, \mathrm{Co}^{2+}, \mathrm{Ni}^{2+}, \mathrm{Cu}^{2+}, \mathrm{Cd}^{2+}, \mathrm{Pb}^{2+}$, and $\mathrm{Hg}^{2+}$ were carried out using neat PSCMS- $g$-DGEBA membrane and PSCMS- $g$-DGEBA/AuNPs-PSNPs 1 membranes. After filtration, the concentration of each cation in the liquid phase was determined by inductively coupled plasma mass spectrometry (ICP-MS, Agilent 7500). Consecutive dilutions of sample aliquots with ultra-pure water/ nitric acid $(5 \% \mathrm{v} / \mathrm{v})$ were performed to reach concentrations in the range of the calibration curve (0-40 ppb), and thus direct information regarding the extraction percentages of metal ions by membranes was obtained. The effectiveness of membranes for the extraction of environmentally toxic heavy metal ions was studied in terms of 
distribution coefficient $\left(\mathrm{Kd}^{\prime}\right)$ and solid-liquid extraction selectivity $\left(\alpha_{\mathrm{S}, \mathrm{L}}\right) \cdot \mathrm{Kd}^{\prime}$ is the measure of the capacity of material to extract cations under competitive conditions, where $\% E$ is extraction percentage, $V$ is volume of the solution (liters), and $n$ the molar amount of polymer structural units.

$\mathrm{Kd}^{\prime}=\left(\frac{\% E}{100-\% E}\right)\left(\frac{V}{n}\right)$,

$\alpha_{\mathrm{S}, \mathrm{L}}$ is the ratio of two distribution coefficients. $\mathrm{Hg}^{2+}$ cation was taken as the reference, as it has the highest distribution coefficient $\left(\mathrm{Kd}^{\prime}{ }_{\mathrm{M} 2}\right)$.

$\alpha_{S, L}=\frac{\mathrm{Kd}_{M 1}}{\mathrm{Kd}_{M 2}}=\frac{\mathrm{Kd}_{M 1}^{\prime}}{\mathrm{Kd}_{M 2}^{\prime}}$

\section{Synthesis of polystyrene microspheres (PSNPs)}

Initially, PVP stabilizer (2.6 g) was dissolved in 2-propanol $(160 \mathrm{~mL})$ at $70{ }^{\circ} \mathrm{C}$. AIBN initiator $(0.2 \mathrm{~g})$ was then dissolved in styrene $(20 \mathrm{~g})$ and added to the above mixture with stirring. The polymerization was allowed to proceed at $70{ }^{\circ} \mathrm{C}$ for $24 \mathrm{~h}$. Subsequently, the PS microspheres were centrifuged and washed with ethanol and deionized water (Wu et al. 2010).

\section{Synthesis of gold nanoparticle-coated polystyrene nanocomposite particles (AuNPs-PSNPs)}

$0.025 \mathrm{~g} \mathrm{Na}_{2} \mathrm{CO}_{3}$ was dissolved in $100 \mathrm{~mL}$ deionized water and added to $16.4 \mathrm{~mL} \mathrm{HAuCl}_{4}$ aqueous solution $\left(3 \times 10^{-3} \mathrm{M}\right)$ and stirred for $0.5 \mathrm{~h}$. Then $0.1 \mathrm{~g}$ PS (10 wt $\%$ ) emulsion and $0.25 \mathrm{~mL}$ formaldehyde (35 wt $\%$ ) were added to the above mixture and stirred for $1 \mathrm{~h}$. The resulting AuNPs-PSNPs nanoparticles were separated out by centrifugation and re-dispersion in ethanol and deionized water to remove the residual free nanoparticles.

\section{Preparation of the hybrid membranes}

For the preparation of membranes, the homogenous solution of poly(styrene-co-chloromethylstyrene) matrix and DGEBA were mixed in the ratio of 90:10 wt $\%$ in $10 \mathrm{~mL}$ DMAc. Afterwards, $1 \mathrm{wt} \%$ of HDA was added as crosslinking agent to the above mixture (Fig. 1). The mixture was refluxed at $80{ }^{\circ} \mathrm{C}$ for $6 \mathrm{~h}$. The desired amount of AuNPs-PSNPs was added and reaction flask was transferred to ultrasonic bath cleaner for $6 \mathrm{~h}$. The homogeneous solutions obtained were cast onto glass plates using casting knife. The prepared films were dipped into deionized water as non-solvent. In this step, the exchange of solvent and non-solvent led to the membrane formation. After finishing phase separation step and membrane formation, the membranes were kept in deionized water for $24 \mathrm{~h}$ to remove remaining solvent. Finally, the membranes were dried at $80{ }^{\circ} \mathrm{C}$ for $24 \mathrm{~h}$ before testing (Buonomenna et al. 2007). The membrane properties were measured using the experimental nanofiltration set-up (Fig. 2). FTIR (KBr, $\mathrm{cm}^{-1}$ ) (Fig. 3): 3401 (O-H stretch), 3254, 1519 (N-H stretch and bend), 3005 (aromatic $\mathrm{C}-\mathrm{H}$ stretch), 2911 (aliphatic C-H stretch), $1420 \mathrm{~cm}^{-1}$ (C-N stretch), 1299 (C-O stretch).

\section{Results and discussion}

\section{Mechanical features}

The PSCMS- $g$-DGEBA graft copolymer was reinforced with gold/polystyrene composite nanoparticles in the range of 0.1-1 wt\%. As shown in Fig. 4 and Table 1, there was improvement in mechanical properties of PSCMS- $g$ DGEBA/AuNPs-PSNPs 0.1-1 membranes. There was an increasing trend in the tensile stress, tangent modulus, and toughness of the membranes, while the elongation at break was decreased in the novel membranes. Consequently, the elongation at break of the PSCMS- $g$-DGEBA/AuNPsPSNPs 0.1-1 membranes was decreased from 4.6 to $3.1 \%$ with the increasing filler content. The tensile strength of unfilled PSCMS- $g$-DGEBA matrix was found to be lower (31.2 MPa) compared with the composite membranes, whereas the membranes filled with AuNPs-PSNPs nanoparticles demonstrated enhancement in the tensile strength from 32.5 to $35.2 \mathrm{MPa}$. In the series, the tensile strength was higher for PSCMS- $g$-DGEBA/AuNPs-PSNPs 1 hybrid with $1 \mathrm{wt} \%$ filler content (35.2 $\mathrm{MPa}$ ) compared with the PSCMS- $g$-DGEBA/AuNPs-PSNPs $0.1-0.5$ membranes (32.5-34.2 MPa) with lower filler amount. The addition of nanoparticles resulted in modest increase in the tensile strength. This may be related to moderately uniform distribution of nanoparticles with their adding amounts. As revealed by the fractured surfaces in Fig. $7 \mathrm{c}$ and $\mathrm{d}$ (PSCMS- $g$-DGEBA/AuNPs-PSNPs 0.1), improvement in the tensile properties was accompanied by a transition from a brittle non-uniform fracture (Fig. 6a) for PSCMS- $g$ DGEBA/AuNPs-PSNPs 1 to uniform patterned structure. Therefore, the mechanism behind the slight improvement in the mechanical properties of the composite was actually reliant on the alteration of the inconsistent fracture surface towards the unvarying structure. Moreover, the higher nanoparticle content actually provided greater interface for the polymer chains, compared with the lower AuNPsPSNPs level. The enhancement was due to the fine miscibility of the particles with the matrix (Ashraf et al. 2014). Furthermore, there was fine enhancement in the tensile modulus for the PSCMS- $g$-DGEBA/AuNPs-PSNPs 1 membrane (2.9 GPa) relative to PSCMS- $g$-DGEBA/ 
Fig. 1 Schematic illustration for the formation of PSCMS- $g$ DGEBA<smiles>CCC(CC(C)C)c1ccc(CCl)cc1</smiles><smiles>CC(C)(c1ccc(OCC2CO2)cc1)c1ccc(OCC2CO2)cc1</smiles><smiles>NCCCCCCN</smiles><smiles>CCCC(C)C(CC)c1ccccc1</smiles>

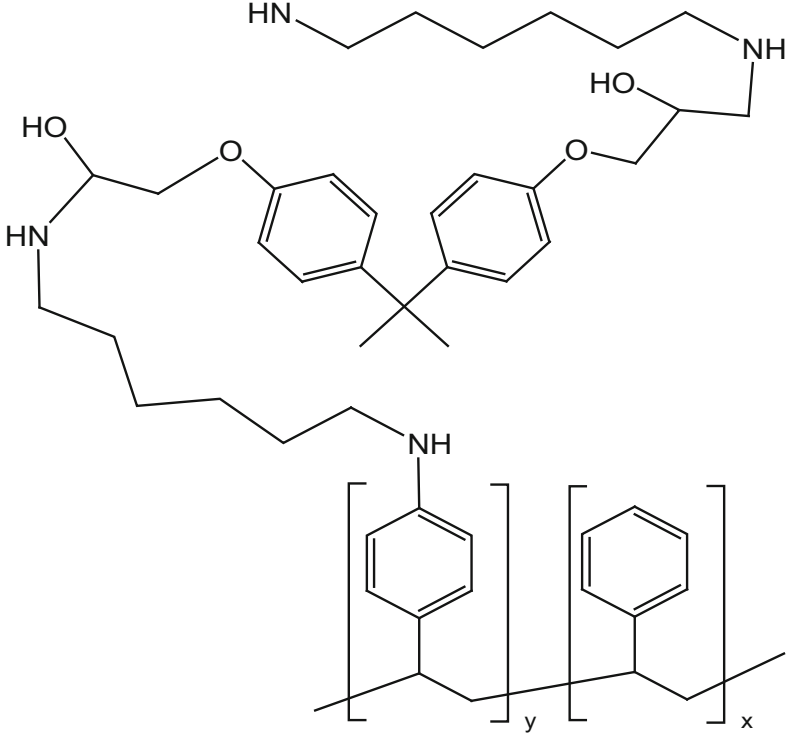

AuNPs-PSNPs 0.1 (1.8 GPa), PSCMS- $g$-DGEBA/AuNPsPSNPs 0.3 (2.2 GPa), and PSCMS- $g$-DGEBA/AuNPsPSNPs 0.5 (2.6 GPa) and neat matrix (1.5 GPa). Moreover, AuNPs-PSNPs caused an improvement in the toughness of the system from 333 to $466 \mathrm{Jm}^{-3}$. Here again the toughness of the composites was found to be higher than the base matrix $\left(78 \mathrm{Jm}^{-3}\right)$. The physical adhesion between the filler particles and the matrix actually operated as a key factor in enhancing the tensile characteristics.

\section{Morphological investigation}

The TEM image of gold nanoparticles has revealed round shape and uniform distribution (Fig. 5a). The AuNPs were also analyzed with Zetasizer Nano ZS particle analyzer and found to have size of $10-20 \mathrm{~nm}$. Figure $5 \mathrm{~b}$ shows the polystyrene microspheres physically embedded with the gold nanoparticles. The AuNPs-PSNPs composite nanoparticles also acquired spherical shape in the size range of 30-50 nm having narrow distribution. Due to high specific surface area, the composite nanoparticles offer ample interphase for the polymer/filler interaction. Thus, the polymer segments aligned with the adjoining filler particles may form well-oriented structure. Accordingly, the inside particulars of the composites turned out to be of higher strength and modulus as explained in the preceding section. The FESEM images of PSCMS-g-DGEBA/ AuNPs-PSNPs 0.1, PSCMS- $g$-DGEBA/AuNPs-PSNPs 0.3, and PSCMS- $g$-DGEBA/AuNPs-PSNPs 0.5 are given in Fig. 6a-e. The PSCMS-g-DGEBA 0.1 with $0.1 \mathrm{wt} \%$ 
Fig. 2 Experimental nanofiltration setup

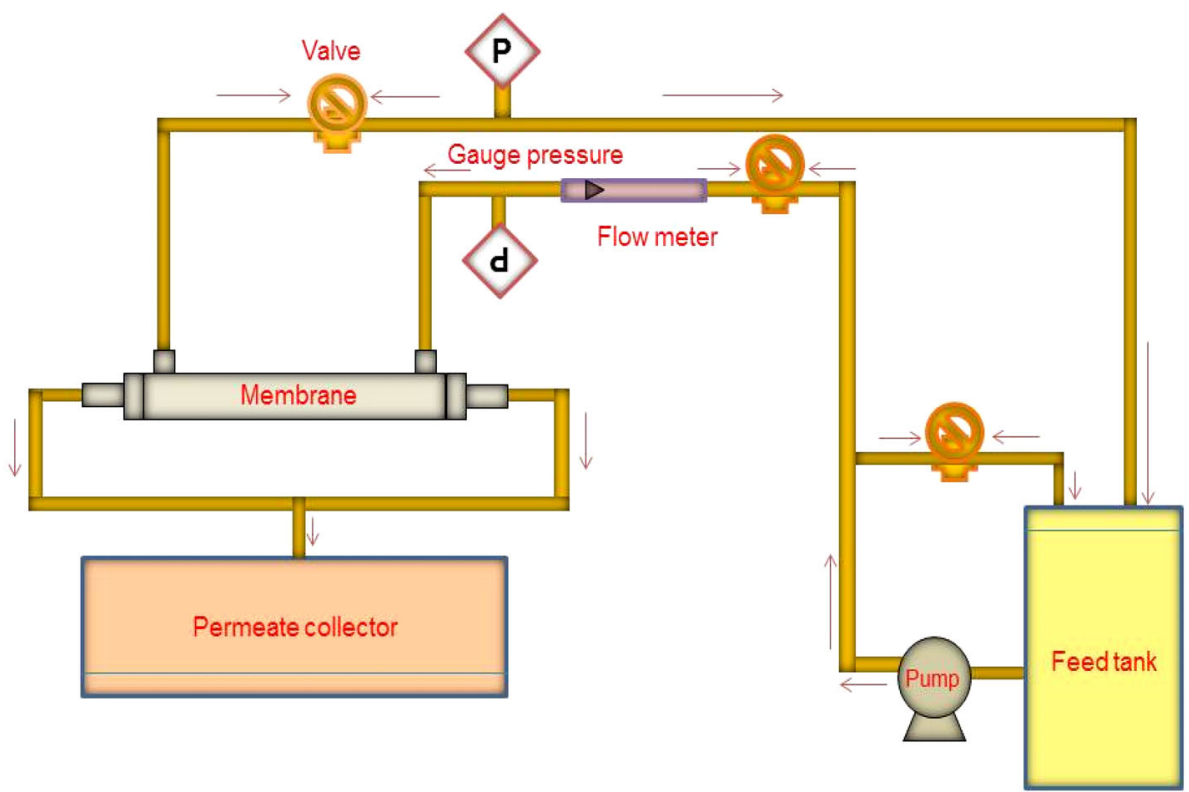

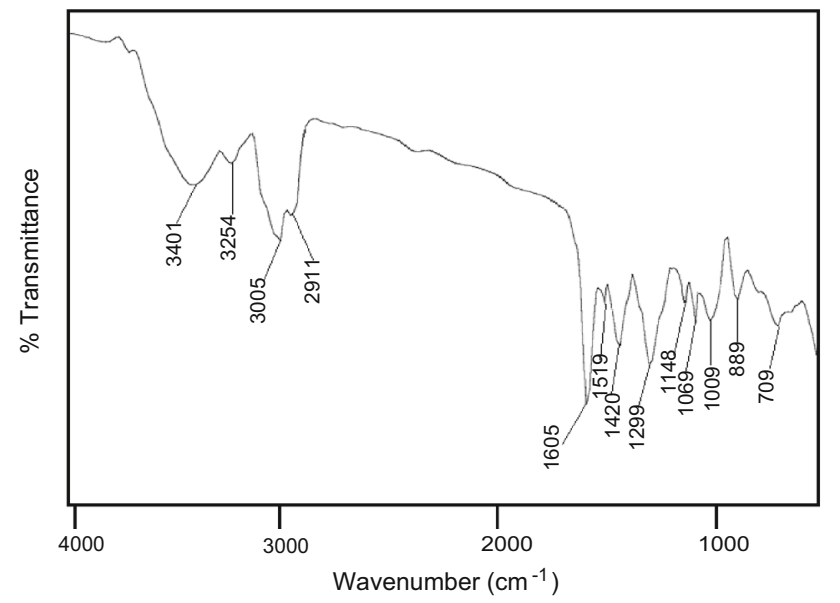

Fig. 3 FTIR spectrum of PSCMS- $g$-DGEBA

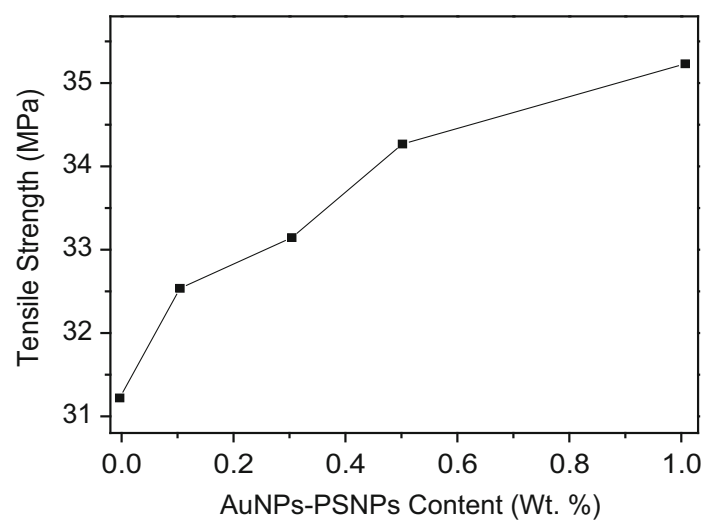

Fig. 4 Drift of tensile strength $v s$. AuNPs-PSNPs in PSCMS- $g$ DGEBA/AuNPs-PSNPs membranes
AuNPs-PSNPs composite nanoparticles revealed porous morphology but the nanopores were not homogeneously dispersed (Fig. 6a-c). The micrograph in Fig. 6d (PSCMS$g$-DGEBA/AuNPs-PSNPs 0.3) showed somewhat regular porous morphology. It appeared that the inclusion of $0.5 \mathrm{wt} \%$ filler formed few circular areas with the uniformly distributed nanopores (Fig. 6e). The cross-section morphology of PSCMS- $g$-DGEBA/AuNPs-PSNPs 1 membrane showed sponge-like micro-pores (Fig. 7a, b). The increase in the nanopores was also obvious in Fig. 7c and d compared with PSCMS- $g$-DGEBA/AuNPs-PSNPs 0.1-0.5. The appropriate amount of membrane porosity is usually important to increase the filtration competence (Jing et al. 2008). On the other hand, the extremely high porosity and larger size pores may unfavorably impact the membrane performance because pores might not be closed effectively and the membrane tends to shrink. The pore size of the membranes was calculated using BET analyzer. There was increase in the pore size of the membranes with the nanoparticle addition. PSCMS- $g$-DGEBA has mean pore size of $50 \mathrm{~nm}$. PSCMS- $g$-DGEBA/AuNPs-PSNPs 0.1 has pore size of $80 \mathrm{~nm}$, PSCMS- $g$-DGEBA/AuNPs-PSNPs 0.3 has pore size of $90 \mathrm{~nm}$, PSCMS- $g$-DGEBA/AuNPs-PSNPs 0.5 has pore size of $100 \mathrm{~nm}$, and PSCMS- $g$-DGEBA/ AuNPs-PSNPs 1 has mean pore size of $120 \mathrm{~nm}$.

\section{Membrane properties and performance}

At constant operating pressure $(410 \mathrm{kPa})$, the pure water flux of PSCMS- $g$-DGEBA and PSCMS- $g$-DGEBA/ AuNPs-PSNPs $0.1-1$ membranes upon compaction was measured every $1 \mathrm{~h}$. During compaction, the water flux was 
Table 1 Mechanical properties of PSCMS- $g$-DGEBA and PSCMS- $g$-DGEBA/AuNPs-PSNPs membranes

\begin{tabular}{llllr}
\hline Membrane type & $\begin{array}{l}\text { Tensile Strength } \\
(\mathrm{MPa}) \pm 0.02\end{array}$ & $\begin{array}{l}\text { Elongation at } \\
\text { break } \pm 0.01(\%)\end{array}$ & $\begin{array}{l}\text { Tensile modulus } \\
(\mathrm{GPa}) \pm 0.03\end{array}$ & $\begin{array}{l}\text { Toughness } \\
\left.(\mathrm{Jm})^{-3}\right) \pm 0.02\end{array}$ \\
\hline PSCMS- $g$-DGEBA & 31.2 & 1.7 & 1.5 & 78 \\
PSCMS- $g$-DGEBA/AuNPs-PSNPs 0.1 & 32.5 & 4.6 & 1.8 & 333 \\
PSCMS- $g$-DGEBA/AuNPs-PSNPs 0.3 & 33.1 & 4.1 & 2.2 & 378 \\
PSCMS- $g$-DGEBA/AuNPs-PSNPs 0.5 & 34.2 & 3.6 & 2.6 & 421 \\
PSCMS- $g$-DGEBA/AuNPs-PSNPs 1 & 35.2 & 3.1 & 2.9 & 466 \\
\hline
\end{tabular}
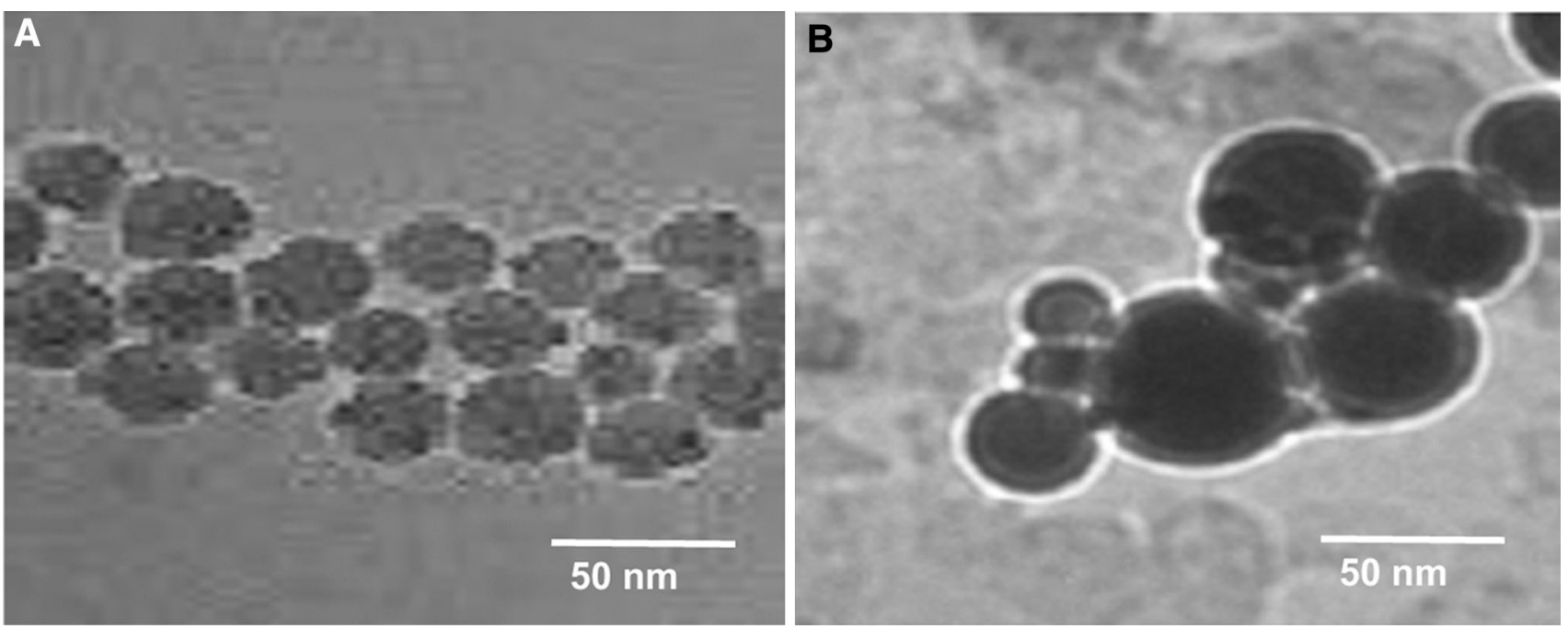

Fig. 5 TEM micrographs of a AuNPs at $50 \mathrm{~nm}$; b AuNPs-PSNPs at $50 \mathrm{~nm}$

high initially, declined gradually, and reached a steady state after 2-3 h of compaction. This preliminary decrease in flux might be due to the fact that the membrane pores are being compacted leading to uniform pore size and steadystate water flux. The new membranes after compaction were subjected to a pressure of $340 \mathrm{kPa}$ for the measurement of pure water flux every $1 \mathrm{~h}$. The porosity, \% solvent content, shrinkage ratio, flux, recovery, and salt rejection tests were performed for the evaluation of membrane properties and performance. The porosity measurement of PSCMS- $g$-DGEBA and PSCMS- $g$-DGEBA/AuNPs-PSNPs 0.1-1 membranes is given in Table 2. The unfilled PSCMS$g$-DGEBA membrane possesses lower porosity $\sim 0.12 \mathrm{~g} /$ $\mathrm{cm}^{2}$ in water and also low values in other solvents. Moreover, there was steady increase in the membrane porosity in different solvents (water, ethanol, methanol, and propanol). This increase was due to enhancement in the hydrophilic nature of the membranes with nanofiller addition. As the hydrophilicity of solvent was decreased (moving from water to propanol), the membrane porous nature was also found to decrease. To assess the hydrophilic nature of the membranes, $\%$ solvent content of membranes was calculated (Table 3 ). The $\%$ solvent content had increasing tendency with composite nanoparticle loading. Both the porosity and $\%$ solvent content were maximum for PSCMS- $g$-DGEBA/AuNPs-PSNPs 1 and were found minimum for neat PSCMS- $g$-DGEBA. Similar to the membrane porosity, the \% solvent content was also observed to decline when moving from water to less polar solvent depending upon the solvent hydrophilicity. The porosity and \% water content of PSCMS- $g$-DGEBA/ AuNPs-PSNPs 1 were calculated as $1.42 \mathrm{~g} / \mathrm{cm}^{2}$ and $3.11 \%$, respectively. Furthermore, the higher porosity of PSCMS- $g$-DGEBA/AuNPs-PSNPs 1 resulted in lower shrinkage ratio of the membrane. Table 4 shows the trend of shrinkage ratio of the membranes. As the AuNPs-PSNPs filler content increased from 0.1 to $1 \mathrm{wt} \%$, the shrinkage ratio was found to decrease rapidly. Moving from neat PSCMS- $g$-DGEBA to PSCMS- $g$-DGEBA/AuNPs-PSNPs 0.1-1 membranes, there was decrease in the shrinkage ratio from 13.2 to 10.1 due to increase in nanoporous nature of the membranes. Generally, the measurement of pure water flux is an important criterion to study the performance of nanofiltration membranes. Owing to the increased membrane porosity, \% solvent content, and hydrophilicity, the flux of the membranes was also supposed to increase. As 

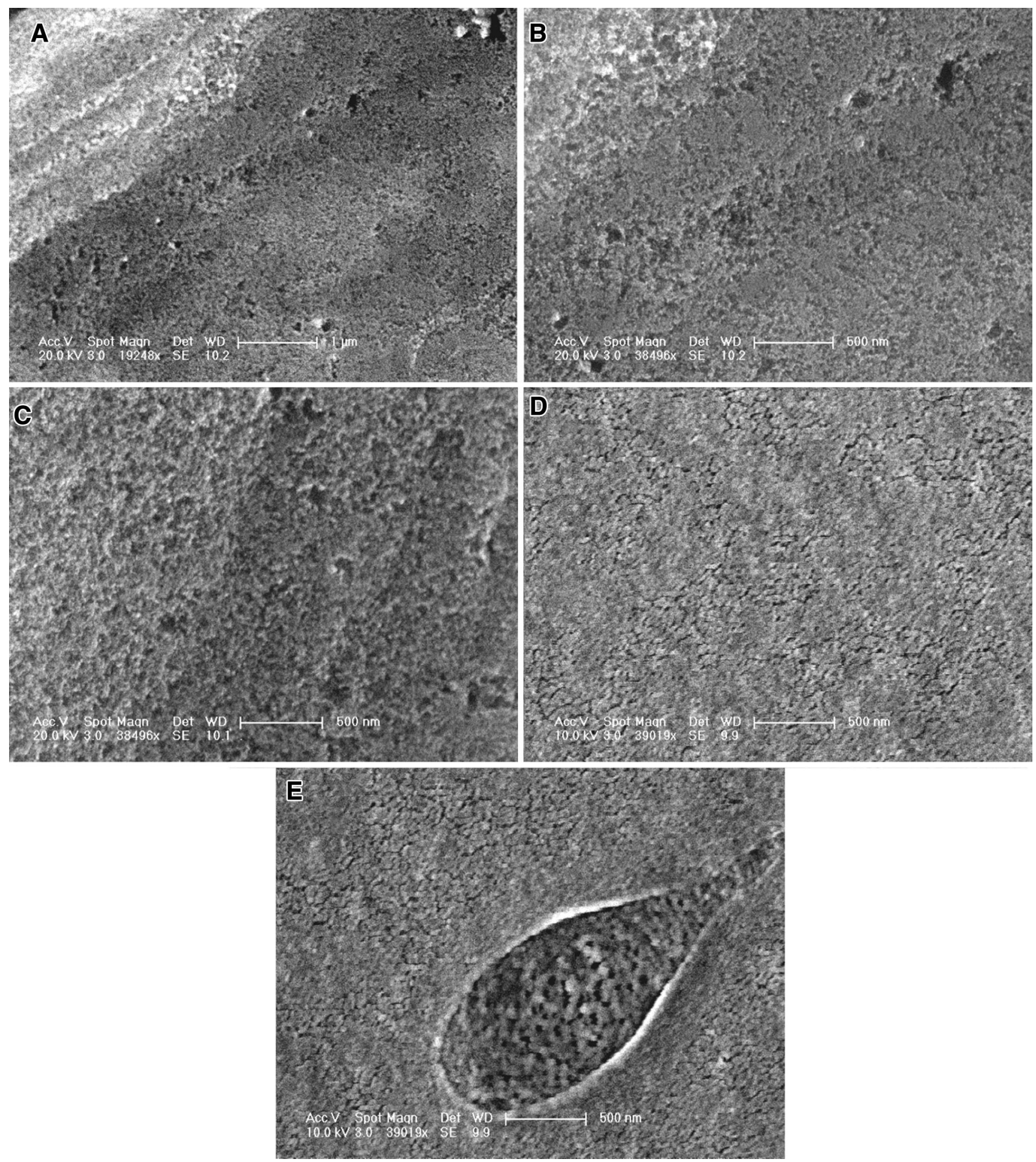

Fig. 6 FESEM micrographs of a PSCMS- $g$-DGEBA/AuNPs-PSNPs 0.1 at $1 \mu \mathrm{m}$; b PSCMS- $g$-DGEBA/AuNPs-PSNPs 0.1 at $500 \mathrm{~nm}$; c PSCMS- $g$-DGEBA/AuNPs-PSNPs 0.1 at $500 \mathrm{~nm}$; d PSCMS- $g$ -

expected, the PSCMS- $g$-DGEBA/AuNPs-PSNPs 1 membrane showed higher flux of $2.01 \mathrm{mLcm}^{-2} \mathrm{~min}^{-1}$ (Table 5). The flux of PSCMS- $g$-DGEBA, PSCMS- $g$ DGEBA/AuNPs-PSNPs 0.1 , PSCMS- $g$-DGEBA/AuNPs-
DGEBA/AuNPs-PSNPs 0.3 at $500 \mathrm{~nm}$; e PSCMS- $g$-DGEBA/AuNPsPSNPs 0.5 at $1 \mu \mathrm{m}$

PSNPs 0.3, and PSCMS- $g$-DGEBA/AuNPs-PSNPs 0.5 membranes was found to be relatively lower as $1.51,1.62$, 1.77, and $1.87 \mathrm{mLcm}^{-2} \mathrm{~min}^{-1}$, respectively. The $\mathrm{NaCl}$ concentration was $35,000 \mathrm{mg} / \mathrm{L}$ and osmotic pressure was 

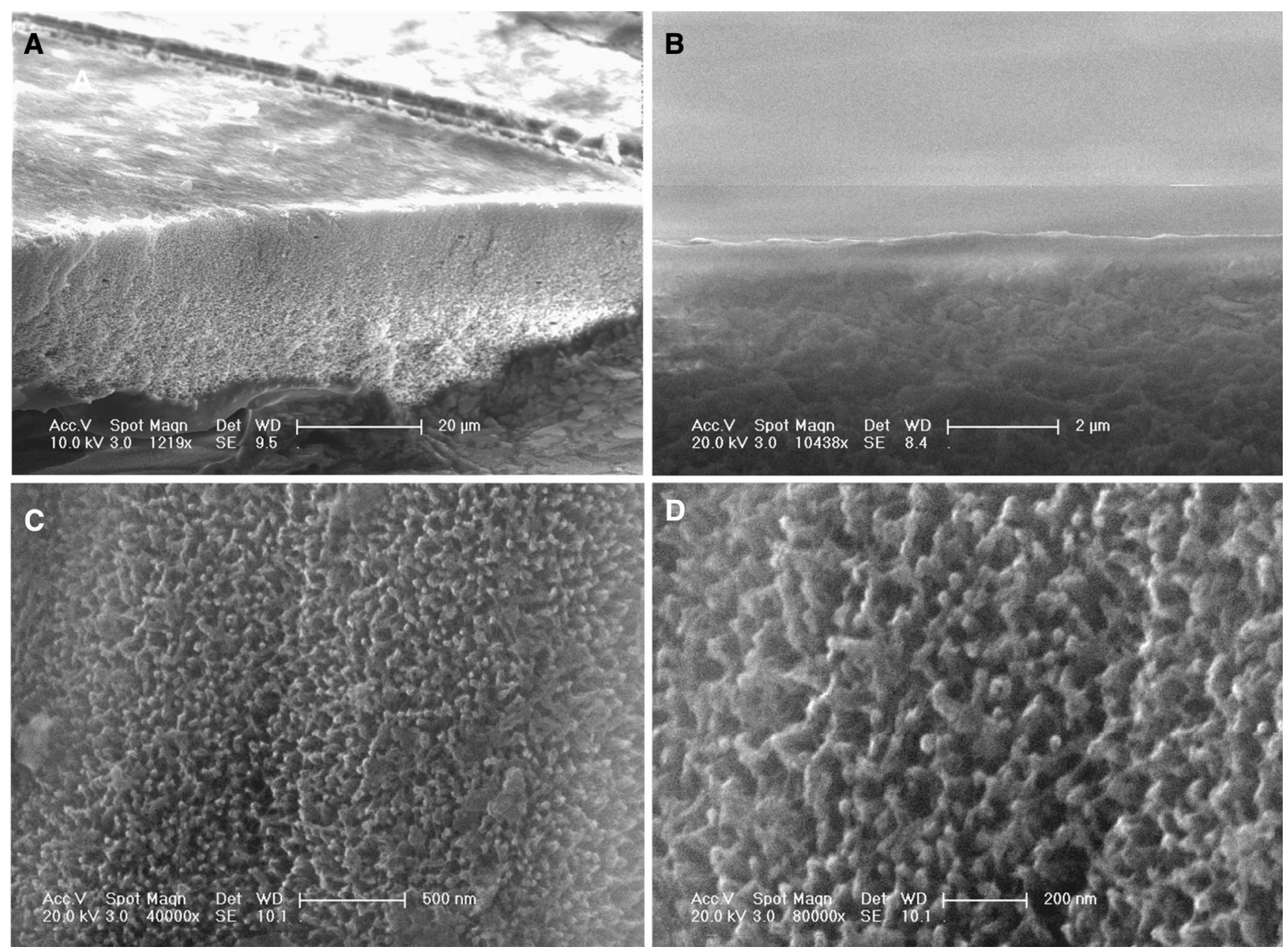

Fig. 7 FESEM micrographs of a PSCMS- $g$-DGEBA/AuNPs-PSNPs 1 at $20 \mu \mathrm{m}$; b PSCMS- $g$-DGEBA/AuNPs-PSNPs 1 at $2 \mu \mathrm{m}$; $\mathbf{c}$ PSCMS- $g$ DGEBA/AuNPs-PSNPs 1 at $500 \mathrm{~nm}$; and d PSCMS- $g$-DGEBA/AuNPs-PSNPs 1 at $200 \mathrm{~nm}$

Table 2 Porosity of PSCMS- $g$ DGEBA and PSCMS- $g$ DGEBA/AuNPs-PSNPs membranes in different solvents

Table $3 \%$ Solvent content of PSCMS- $g$-DGEBA and PSCMS- $g$-DGEBA/AuNPsPSNPs membranes in different solvents

\begin{tabular}{lllll}
\hline Membrane type & \multicolumn{2}{l}{ Porosity $\left(\mathrm{g} / \mathrm{cm}^{2}\right)$} & \\
\cline { 2 - 5 } & Water & Ethanol & Methanol & Propanol \\
\hline PSCMS- $g$-DGEBA & 0.12 & 0.11 & 0.07 & 0.05 \\
PSCMS- $g$-DGEBA/AuNPs-PSNPs 0.1 & 0.56 & 0.43 & 0.13 & 0.07 \\
PSCMS- $g$-DGEBA/AuNPs-PSNPs 0.3 & 0.69 & 0.55 & 0.32 & 0.09 \\
PSCMS- $g$-DGEBA/AuNPs-PSNPs 0.5 & 0.98 & 0.67 & 0.39 & 0.13 \\
PSCMS- $g$-DGEBA/AuNPs-PSNPs 1 & 1.42 & 0.91 & 0.45 & 0.19 \\
\hline
\end{tabular}

\begin{tabular}{lllll}
\hline Membrane type & Water & Ethanol & Methanol & Propanol \\
\hline PSCMS- $g$-DGEBA & 2.11 & 1.23 & 0.99 & 0.78 \\
PSCMS- $g$-DGEBA/AuNPs-PSNPs 0.1 & 2.43 & 1.32 & 1.12 & 0.87 \\
PSCMS- $g$-DGEBA/AuNPs-PSNPs 0.3 & 2.65 & 1.45 & 1.22 & 0.98 \\
PSCMS- $g$-DGEBA/AuNPs-PSNPs 0.5 & 2.76 & 1.77 & 1.32 & 1.17 \\
PSCMS- $g$-DGEBA/AuNPs-PSNPs 1 & 3.11 & 1.89 & 1.43 & 1.26 \\
\hline
\end{tabular}

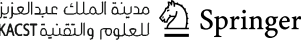


Table 4 Shrinkage ratio of PSCMS- $g$-DGEBA and PSCMS- $g$-DGEBA/AuNPsPSNPs membranes in different solvents

\begin{tabular}{lllll}
\hline Membrane type & Water & Ethanol & Methanol & Propanol \\
\hline PSCMS- $g$-DGEBA & 13.2 & 9.15 & 8.21 & 7.12 \\
PSCMS- $g$-DGEBA/AuNPs-PSNPs 0.1 & 12.9 & 8.31 & 8.14 & 6.57 \\
PSCMS- $g$-DGEBA/AuNPs-PSNPs 0.3 & 12.1 & 7.98 & 7.97 & 6.44 \\
PSCMS- $g$-DGEBA/AuNPs-PSNPs 0.5 & 10.6 & 7.52 & 7.16 & 5.93 \\
PSCMS- $g$-DGEBA/AuNPs-PSNPs 1 & 10.1 & 7.1 & 6.99 & 5.33 \\
\hline
\end{tabular}

\begin{tabular}{llll}
\hline Membrane type & $\begin{array}{l}\text { Pure water flux } \\
\left(\mathrm{mL} \mathrm{cm} \mathrm{min}^{-1}\right)^{\mathrm{a}}\end{array}$ & $\begin{array}{l}\text { Salt rejection } \\
(\%)\end{array}$ & $\begin{array}{l}\text { Recovery } \\
(\%)\end{array}$ \\
\hline PSCMS- $g$-DGEBA & 1.51 & 52.6 & 71.2 \\
PSCMS- $g$-DGEBA/AuNPs-PSNPs 0.1 & 1.62 & 58.1 & 75.1 \\
PSCMS- $g$-DGEBA/AuNPs-PSNPs 0.3 & 1.77 & 62.4 & 78.5 \\
PSCMS- $g$-DGEBA/AuNPs-PSNPs 0.5 & 1.87 & 65.3 & 80.6 \\
PSCMS- $g$-DGEBA/AuNPs-PSNPs 1 & 2.01 & 70.4 & 83.8 \\
\hline
\end{tabular}

${ }^{\text {a }}$ Applied pressure $=50 \mathrm{bar}$
Table 5 Pure water flux, salt rejection, recovery of PSCMS$g$-DGEBA and PSCMS- $g$ DGEBA/AuNPs-PSNPs membranes other side of the membrane as permeate (He et al. 2011). If the pore size is larger then the salt solution will flow in the central region of the pore contaminating the permeate. So the mechanism for the desalination from such membranes is based on the presence of the nanopores. Similar mechanism was operative for the removal of other contaminants from the polluted water. Second, membranes containing higher amount of gold nanoparticles become more porous and macrovoids are diminishing at higher concentrations. Therefore, the denser membranes revealed intrinsically higher flux (Vanherck et al. 2011; Cobley and Xia 2009). The membrane stability was also investigated in terms of the persistence of nanoparticles inside the membrane structure during filtration process. The composite nanoparticles of gold were covered with the polystyrene shell. Moreover, these nanoparticles were reinforced inside the epoxy blend matrix and cannot be easily released into the filtrate. For the confirmation, permeate was tested for the presence of gold nanoparticles. The occurrence of gold nanoparticles in permeate was determined using UV-VIS technique. The solution was characterized by a Perkin Elmer UV-VIS spectrophotometer for the typical large peak at $530 \mathrm{~nm}$, corresponding to the plasmon absorbance band of the AuNPs. A strong peak at $530 \mathrm{~nm}$ was not observed in the UV-VIS spectrum indicating the absence of the nanoparticles in the filtrate. This means that the AuNPs did not turn into the secondary pollution during water purification.

\section{Extraction of toxic metal ions}

In PSCMS- $g$-DGEBA, the thiourea moieties were introduced in the matrix backbone. Thiourea-based polymers have been reported for the extraction of toxic metal ions 
Table 6 Solid-liquid extraction of various metal cations from aqueous solution by PSCMS- $g$-DGEBA and PSCMS- $g$-DGEBA/AuNPs-PSNPs 1

\begin{tabular}{|c|c|c|c|c|c|c|}
\hline \multirow[t]{2}{*}{ Salt } & \multicolumn{2}{|l|}{$\% E$} & \multicolumn{2}{|c|}{$\mathrm{Kd}^{\prime} / \mathrm{L} \mathrm{mol}^{-1}$} & \multicolumn{2}{|l|}{$\alpha \times 10^{3}$} \\
\hline & $\begin{array}{l}\text { PSCMS- } g- \\
\text { DGEBA }\end{array}$ & $\begin{array}{l}\text { PSCMS- } g \text {-DGEBA/ } \\
\text { AuNPs-PSNPs } 1\end{array}$ & $\begin{array}{l}\text { PSCMS- } g- \\
\text { DGEBA }\end{array}$ & $\begin{array}{l}\text { PSCMS- } g \text {-DGEBA/ } \\
\text { AuNPs-PSNPs } 1\end{array}$ & $\begin{array}{l}\text { PSCMS- } g \text { - } \\
\text { DGEBA }\end{array}$ & $\begin{array}{l}\text { PSCMS- } g \text {-DGEBA/ } \\
\text { AuNPs-PSNPs } 1\end{array}$ \\
\hline $\mathrm{Fe}\left(\mathrm{NO}_{3}\right)_{3}$ & 35 & 38 & 18 & 29 & 8 & 9 \\
\hline $\mathrm{Cu}\left(\mathrm{NO}_{3}\right)_{2}$ & 36 & 68 & 20 & 95 & 9 & $1 \times 10^{1}$ \\
\hline $\mathrm{Co}\left(\mathrm{NO}_{3}\right)_{2}$ & 36 & 68 & 20 & 95 & 9 & $1 \times 10^{1}$ \\
\hline $\mathrm{Cd}\left(\mathrm{NO}_{3}\right)_{2}$ & 36 & 68 & 20 & 95 & 9 & $1 \times 10^{1}$ \\
\hline $\mathrm{Ni}\left(\mathrm{NO}_{3}\right)_{2}$ & 36 & 68 & 20 & 95 & 9 & $1 \times 10^{1}$ \\
\hline $\mathrm{Pb}\left(\mathrm{NO}_{3}\right)_{2}$ & 95 & 99 & $30 \times 10^{2}$ & $30 \times 10^{2}$ & $1 \times 10^{3}$ & $1 \times 10^{3}$ \\
\hline $\mathrm{Hg}\left(\mathrm{NO}_{3}\right)_{2}$ & 95 & 99 & $30 \times 10^{2}$ & $30 \times 10^{2}$ & $1 \times 10^{3}$ & $1 \times 10^{3}$ \\
\hline
\end{tabular}

from water (Kausar and Hussain 2013a, b). The PSCMS- $g$ DGEBA/AuNPs-PSNPs membranes were tested in terms of distribution coefficient $\left(\mathrm{Kd}^{\prime}\right)$ and solid-liquid extraction selectivity $\left(\alpha_{S, L}\right)$ (Duhart et al. 2001). Table 6 shows the results obtained for the extraction of toxic metal ions using neat PSCMS- $g$-DGEBA and PSCMS- $g$-DGEBA/AuNPsPSNPs 1 membranes. The extraction percentage for $\mathrm{Fe}^{3+}$, $\mathrm{Co}^{2+}, \mathrm{Ni}^{2+}, \mathrm{Cu}^{2+}$, and $\mathrm{Cd}^{2+}$ was $68 \%$ in the case of PSCMS- $g$-DGEBA/AuNPs-PSNPs 1 . However, the efficiency of the membranes was exceptional for $\mathrm{Pb}^{2+}$ and $\mathrm{Hg}^{2+}$, i.e., the screening extraction level was close to $100 \%$ from aqueous solution. The higher extraction percentage, towards the elimination of heavy metal ions, was due to the combined affect of thiourea moieties and gold nanoparticles (Calderón et al. 2007). Particularly, high extraction level of lead and mercury shows the potential of novel selective ion transport membranes for decontamination of water.

\section{Analysis of glass transition temperature}

Differential scanning calorimetry was used to study the glass transition temperature of the membranes. The neat PSCMS- $g$-DGEBA matrix revealed glass transition temperature of $123{ }^{\circ} \mathrm{C}$ which is sufficiently higher due to to the cross-linked structure. As shown in Fig. 8, the glass-transition temperature of pure matrix was further increased with the addition of AuNPs-PSNPs. The results depicted that the polymer-based composite nanoparticles developed interaction with the matrix to further enhance its crosslinking structure. The segmental $T_{\mathrm{g}}$ of PSCMS- $g$ DGEBA/AuNPs-PSNPs 0.1-1 was recorded in the range of $132-159{ }^{\circ} \mathrm{C}$. The PSCMS- $g$-DGEBA/AuNPs-PSNPs 0.1 , PSCMS- $g$-DGEBA/AuNPs-PSNPs $0.3, \quad$ PSCMS- $g$ DGEBA/AuNPs-PSNPs 0.5, and PSCMS- $g$-DGEBA/ AuNPs-PSNPs 1 membranes possess glass transition temperature of $132,137,140$, and $159{ }^{\circ} \mathrm{C}$, respectively. Consequently, the DSC curves showed clear shift towards higher temperature with the inclusion of nanofiller. The

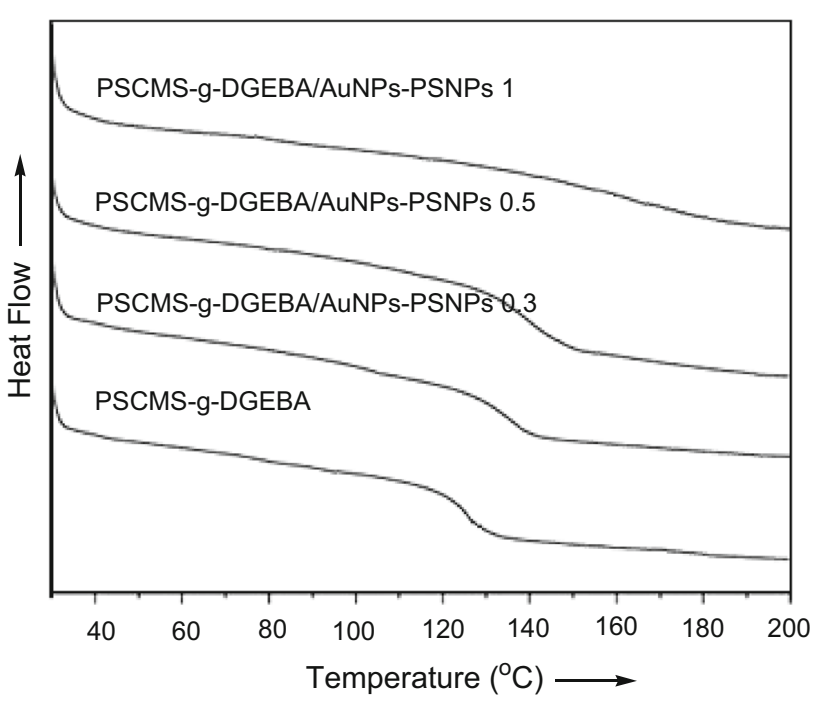

Fig. 8 DSC curves of PSCMS- $g$-DGEBA with varied AuNPs-PSNPs content

increase in the glass transition for the system depicted the increase in the structural rigidity of PSCMS- $g$-DGEBA due to better interaction with the filler.

\section{Conclusions}

The epoxy grafted poly(styrene-co-chloromethylstyrene) was prepared using diamine linker and solution polymerization technique. The spherical gold/polystyrene composite nanoparticles having size of $30-50 \mathrm{~nm}$ were designed for the reinforcement of the membranes. The composite nanoparticles were loaded in varying concentration of $0.1-1 \mathrm{wt} \%$. The mechanical behavior of the AuNPs-PSNPs filled membranes was found to improve with nanoparticle loading. Predominantly in $1 \mathrm{wt} \%$ loaded PSCMS- $g$-DGEBA/AuNPs-PSNPs 1, the cross-linked polystyrene/epoxy showed fine compatibility and strong interactions with the composite nanoparticles, thus 
resulting in adequately enhanced mechanical properties. Another unique advantage of the composite nanofiller was the increase in glass transition temperature of the membranes compared with the pure matrix. In surface morphology analysis, the entire embedding character of nanoparticles in the nanoporous membrane was observed. Moreover, the PSCMS- $g$-DGEBA/AuNPs-PSNPs 1 membrane exhibited fine behavior in the removal of $\mathrm{Pb}^{2+}$ and $\mathrm{Hg}^{2+}$ from water. Study of membrane characteristics such as porosity, flux, shrinkage ratio, recovery, and rejection revealed that the gold/polystyrene composite nanoparticles reinforced novel hybrids are well suited for water purification set-up.

Open Access This article is distributed under the terms of the Creative Commons Attribution 4.0 International License (http:// creativecommons.org/licenses/by/4.0/), which permits unrestricted use, distribution, and reproduction in any medium, provided you give appropriate credit to the original author(s) and the source, provide a link to the Creative Commons license, and indicate if changes were made.

\section{References}

Arockiasamy DL, Alam J, Alhoshan M (2013) Carbon nanotubesblended poly(phenylene sulfone) membranes for ultrafiltration applications. Appl Water Sci 3:93-103

Ashraf R, Kausar A, Siddiq M (2014) High-performance polymer/nanodiamond composites: synthesis and properties. Iran Polym J 23:531-545

Asyhar R (2014) Simple method for the removal of mercury(II) from its aqueous solution using aluminum as a reducing agent. Chem Mater Res 6:2224-3224

Bowen WR, Mohammad AW (1998) Diafiltration by nanofiltration: prediction and optimization. AIChE J 44:1799-1812

Bowen WR, Welfoot JS (2002) Modelling the performance of membrane nanofiltration-critical assessment and model development. Chem Engineer Sci 57:1121-1137

Bowen WR, Mohammad AW, Hilal N (1997) Characterisation of nanofiltration membranes for predictive purposes - use of salts, uncharged solutes and atomic force microscopy. J Membr Sci 126:91-105

Buonomenna MG, Macchib P, Davolic M, Driolia E (2007) Poly(vinylidene fluoride) membranes by phase inversion: the role the casting and coagulation conditions play in their morphology, crystalline structure and properties. Eur Polym J 43:1557-1572

Calderón V, Serna F, García F, de la Peña JL, García JM (2007) Selective solid-liquid extraction of cations using solid-phase polyamides with crown ether moieties as cation host units. J Appl Polym Sci 106:2875-2884

Chen X, Yang H, Gu Z, Shao Z (2001) Preparation and characterization of HY zeolite-filled chitosan membranes for pervaporation separation. J Appl Polym Sci 79:1144-1149

Cobley CM, Xia YN (2009) Gold and nanotechnology. Elements 5:309-313

Coulston Roger J, Jones Samuel T, Lee Tung-Chun, Appel Eric A, Oren A (2011) Scherman. Supramolecular gold nanoparticlepolymer composites formed in water with cucurbit[8]uril. Chem Commun 47:164-166
Dalwani M, Benes NE, Bargeman G, Stamatialis D, Wessling M (2010) A method for characterizing membranes during nanofiltration at extreme $\mathrm{pH}$. J Membr Sci 363:188-194

Du NUL, Abu AB, Azahari B, Ariff ZM, Chujo Y (2011) Porous epoxy microparticles prepared by an advanced aqueous method. Mater Lett 65:1655-1658

Duhart A, Dozol JF, Rouquette H, Deratani A (2001) Selective removal of cesium from model nuclear waste solutions using a solid membrane composed of an unsymmetrical calix[4]arenebiscrown- 6 bonded to an immobilized polysiloxane backbone. J Membr Sci 185:145-155

Ebert K, Fritsch D, Koll J, Tjahjawiguna C (2004) Influence of inorganic fillers on the compaction behaviour of porous polymer based membranes. J Membr Sci 233:71-78

Evuti AM, Lawal M (2011) Recovery of coagulants from water works sludge: a review. Adv Appl Sci Res 2:410-417

Gevers LEM, Vankelecom IFJ, Jacobs PA (2005) Zeolite filled polydimethylsiloxane (PDMS) as an improved membrane for solventresistant nanofiltration (SRNF). Chem Commun 19:2500-2502

Greenlee LF, Lawler DF, Freeman BD, Marrot B, Moulin P (2009) Reverse osmosis desalination: water sources, technology, and today's challenges. Water Res 43:2317-2348

He J, Lin X-M, Chan H, Vukovi L, Kral P, Jaeger HM (2011) Diffusion and filtration properties of self-assembled gold. nanocrystal membranes. Nano Lett 11:2430-2435

Henglein A (1998) Colloidal silver nanoparticles: photochemical preparation and interaction with $\mathrm{O}_{2}, \mathrm{CCl}_{4}$, and some metal ions. Chem Mater 10:444-450

Jing M, Lingling L, Guohua C, Congjie G, Shengxiong D (2008) Preparation of $\mathrm{N}, \mathrm{O}$-carboxymethyl chitosan (NOCC) composite nanofiltration membranes and its rejection performance for the fermentation effluent from a wine factory. J Chem Eng 16:209-213

Kausar A, Hussain ST (2013a) New generation of thermally stable and conducting poly(azomethine-ester)s: nano-blend formation with polyaniline. Polym Int 62:1442-1450

Kausar A, Hussain ST (2013b) Synthesis and properties of poly(thiourea-azo-naphthyl)/multi-walled carbon nanotube composites. J Plast Film Sheet 30:6-27

Kumar R, Sharma K, Tiwary KP, Sen G (2013) Polymethacrylic acid grafted psyllium (Psy-g-PMA): a novel material for waste water treatment. Appl Water Sci 3:285-291

Li Y, Pan Y, Zhu L, Wang Z, Su D, Xue G (2011) Facile and controlled fabrication of functional gold nanoparticle-coated polystyrene composite particle. Macromol Rapid Commun 32:1741-1747

Lu Y, Liu J (2007) Smart nanomaterials inspired by biology: dynamic assembly of error-free nanomaterials in response to multiple chemical and biological stimuli. Acc Chem Res 40:315-323

Malaisamy R, Bruening ML (2005) High-flux nanofiltration membranes prepared by adsorption of multilayer polyelectrolyte membranes on polymeric supports. Langmuir 21:10587-10592

Mehwish N, Kausar A, Siddiq M (2014) Advances in polymer-based nano-structured membranes for water treatment. Polym Plast Technol Engineer 53:1290-1316

Namasivayam C, Periasamy K (1993) Carbon for mercury(I1) removal from aqueous solution. Water Res 27:1663-1668

Ono A, Togashi H (2004) Highly selective oligonucleotide-based sensor for mercury(II) in aqueous solutions. Angew Chem Int Ed 43:4300-4302

Patel NG, Kumar A, Jayawardana VN, Woodworth CD, Yuya PA (2014) Fabrication, nanomechanical characterization, and cytocompatibility of gold-reinforced chitosan bio-nanocomposites. Mater Sci Eng C Mater Biol Appl 44:336-344 
Sadeghi M, Khanbabaei G, Dehaghani AHS, Sadeghi M, Aravand MA, Akbarzade M, Khatti S (2008) Gas permeation properties of ethylene vinyl acetate-silica nanocomposite membranes. J Membr Sci 322:423-428

Slocik JM, Zabinski JS Jr, Phillips DM, Naik RR (2008) Colorimetric response of peptide-functionalized gold nanoparticles to metal ions. Small 4:548-551

Susrutha B, Ram S, Tyagi AK (2012) Effects of gold nanoparticles on rheology of nanofluids containing poly(vinylidene fluoride) molecules. J Nanofluids 1:120-127

Van der Bruggen B, Geens J, Vandecasteele C (2002) Fluxes and rejections for nanofiltration with solvent stable polymeric membranes in water, ethanol and n-hexane. Chem Engineer Sci 57:2511-2518
Vanherck K, Vankelecoma I, Verbiest T (2011) Improving fluxes of polyimide membranes containing gold nanoparticles. J Membr Sci 373:5-13

Vu DQ, Koros WJ, Miller SJ (2003) Mixed matrix membranes using carbon molecular sieves:I. Preparation and experimental results. J Membr Sci 211:311-334

Wu Y, Zhang Y, Xu J, Chen M, Wu L (2010) One-step preparation of $\mathrm{PS} / \mathrm{TiO}_{2}$ nanocomposite particles via miniemulsion polymerization. J Colloid Interface Sci 343:18-24

Yuan W, Zydney AL (2000) Humic acid fouling during ultrafiltration. Environ Sci Technol 34:5043-5050 